\title{
Perinatal Mental Illness and Risk of Incident Autoimmune Disease: A Population-Based Propensity-Score Matched Cohort Study
}

\author{
Hilary K Brown (iD ${ }^{1-5}$ \\ Andrew Wilton ${ }^{5}$ \\ Ning Liu ${ }^{5}$ \\ Joel G Ray ${ }^{5,6}$ \\ Cindy-Lee Dennis (iD ${ }^{6,7}$ \\ Simone $\mathrm{N}$ Vigod (D) $^{3-5}$ \\ 'Department of Health \& Society, \\ University of Toronto Scarborough, \\ Toronto, ON, Canada; ${ }^{2}$ Dalla Lana School \\ of Public Health, University of Toronto, \\ Toronto, ON, Canada; ${ }^{3}$ Department of \\ Psychiatry, University of Toronto, \\ Toronto, ON, Canada; ${ }^{4}$ Women's College \\ Research Institute, Women's College \\ Hospital, Toronto, ON, Canada; ${ }^{5}$ ICES, \\ Toronto, ON, Canada; ${ }^{6} \mathrm{Li}$ Ka Shing \\ Knowledge Institute, St. Michael's \\ Hospital, Toronto, ON, Canada; \\ ${ }^{7}$ Lawrence S. Bloomberg Faculty of \\ Nursing, University of Toronto, Toronto, \\ ON, Canada
}

Background: Studies have demonstrated elevated risk for autoimmune disease associated with perinatal mental illness, but the extent to which this risk is specific to mental illness arising perinatally, and not mental illness generally, is unknown. Our objective was to compare the risk of autoimmune disease in women with mental illness arising within the perinatal period to (1) women with mental illness arising outside the perinatal period and (2) women who did not develop mental illness.

Methods: We conducted a population-based matched cohort study of women aged 15-49 years with no history of mental illness or autoimmune disease in Ontario, Canada, 19982018. The exposed, 60,701 women with mental illness arising between conception and 365 days postpartum were propensity score-matched to (1) 264,864 women with mental illness arising non-perinatally and (2) 469,164 women who did not develop mental illness. Hazard ratios (HR) for autoimmune disease were generated using Cox proportional hazards models. Results: The incidence of autoimmune disease was similar among women with mental illness arising perinatally compared to those with mental illness arising non-perinatally (138.4 vs 140.7 per 100,000 person-years; HR 0.98, 95\% CI 0.92-1.05), and elevated among women with mental illness arising perinatally compared to those who did not develop mental illness (138.4 vs 88.9 per 100,000 person-years; HR 1.54, 95\% CI 1.44-1.64). The HR for the latter comparison was more pronounced for autoimmune disease with brainreactive antibodies than other autoimmune disease.

Conclusion: Perinatal mental illness is associated with increased risk of autoimmune disease that is no different than that of mental illness arising non-perinatally. Women with mental illness, regardless of the timing of onset, could benefit from early detection of autoimmune disease.

Keywords: autoimmune diseases, cohort studies, mental disorders, women's health

\section{Plain Language Summary}

Perinatal mental illness describes a mood or anxiety, psychotic, or other mental disorder arising in pregnancy or the postpartum period. There is preliminary evidence that women with perinatal mental illness are at elevated risk for autoimmune disease. However, the extent to which this risk is specific to mental illness arising in pregnancy or the postpartum period, and not mental illness generally, is unknown. We conducted a study of all women in Ontario, Canada, followed between 1998 and 2018 using medical records databases. The study groups included women with mental illness arising in pregnancy or the postpartum period, women with mental illness arising outside the perinatal period, and women without mental illness. Women were followed until a diagnosis of autoimmune disease, death, emigration, or the end
Correspondence: Hilary K Brown Department of Health \& Society, University of Toronto Scarborough, 1265 Military Trail, Toronto, ON, MIC IA4, Canada

Tel +l 4162082239

Email hk.brown@utoronto.ca 
of the study. We found that women with mental illness arising in pregnancy or the postpartum period were at elevated risk of autoimmune disease compared to those without a mental illness. No differences were observed between women with mental illness arising in pregnancy or the postpartum period and women with mental illness arising outside the perinatal period. These findings show that women with mental illness, regardless of the timing of onset, could benefit from early detection of autoimmune disease.

\section{Introduction}

Perinatal mental illness, which describes a mood or anxiety disorder, psychotic disorder, or other mental illness arising in pregnancy or in the year following delivery, is a frequent complication of childbirth, affecting one in five women. ${ }^{1}$ Perinatal mental illness can have lasting adverse outcomes, with significant societal costs that often include intergenerational effects. ${ }^{2,3}$ While women with perinatal mental illness are at increased risk for chronic mental illness, ${ }^{2}$ little is known about the impact of perinatal mental illness on the risk of chronic physical conditions. Such data could provide important insight into the underlying pathophysiology of perinatal mental illness and lead to the development of interventions to mitigate long-term risks associated with perinatal mental illness.

There is preliminary evidence that women with perinatal mental illness are at elevated risk for autoimmune disease. ${ }^{4,5}$ Some authors have postulated that perinatal mental illness is itself an autoimmune disease. ${ }^{6}$ Autoimmune diseases are a clinically heterogenous group of disorders defined by an abnormal immunologic reaction to self-antigens resulting in tissue damage and dysfunction of specific organs or entire body systems, including the endocrine, gastrointestinal, liver, neurological, and rheumatological systems. ${ }^{7}$ Like many other autoimmune diseases that are affected by the maternal immunologic response to implantation and delivery, ${ }^{8}$ perinatal mental illness flares in early pregnancy and early postpartum. Immune-mediated cytokine alterations have also been found in women with postpartum depression. ${ }^{9,10}$ Yet, the extent to which autoimmune disease risk is related to mental illness arising in the perinatal period specifically is unknown. Studies of the association between perinatal mental illness and autoimmune disease have not considered if women with perinatal and non-perinatal mental illness are differentially vulnerable to autoimmune disease. ${ }^{4,5}$ Outside of pregnancy, adults with mental illness are at elevated risk of autoimmune diseases, ${ }^{11}$ particularly those with brain-reactive antibodies, ${ }^{12}$ with mechanisms possibly linked to the sympathetic nervous system and hypothalamic-pituitary-adrenal axis, which regulate immune functioning. ${ }^{13,14}$

Our objective was to determine whether perinatal mental illness is uniquely associated with the development of incident autoimmune disease. We addressed this objective by comparing the risk of incident autoimmune disease among women with mental illness arising in the perinatal period to the risk of incident autoimmune disease in women with mental illness arising outside the perinatal period, and women who did not develop mental illness.

\section{Materials and Methods Study Design and Data Sources}

We conducted a population-based matched cohort study in Ontario, Canada, where all 14.7 million residents are enrolled in a health care plan that covers medically necessary care. We accessed and analyzed data at ICES (Toronto), an independent, non-profit organization that holds administrative datasets derived from residents' health care encounters and linked using a unique encoded identifier: the Canadian Institute for Health Information Discharge Abstract Database for hospitalizations, including birth records from the MOMBABY dataset; Ontario Mental Health Reporting System for psychiatric hospitalizations; National Ambulatory Care Reporting System for emergency department visits; Ontario Health Insurance Plan database for physician visits; and Registered Persons Database for dates of birth and death and postal code. ICES datasets have been shown to have good completeness, with $<3 \%$ missingness, and accuracy, with primary diagnoses showing $74 \%$ to $96 \%$ agreement with chart reabstraction. ${ }^{15}$ Data use was authorized under section 45 of Ontario's Personal Health Information Protection Act, which does not require Research Ethics Board review.

\section{Study Population}

We identified all women alive in Ontario between April 1, 1998 and March 31, 2018. Mental illness was defined as a diagnosis of a mood or anxiety disorder, psychotic disorder, or other mental illness in $\geq 2$ visits with a psychiatrist or general practitioner or $\geq 1$ emergency department visits or hospitalizations (Table S1). We first excluded women with evidence of mental illness before April 1, 1998. Women were then assigned a study index 
date as follows: If a new mental illness diagnosis occurred within a perinatal period (ie, between conception and 365 days postpartum for livebirths or stillbirths), ${ }^{16}$ women were classified as exposed, and their index date was their diagnosis date. If a new mental illness diagnosis occurred outside a perinatal period, they were classified as the first comparison group; their index date was their diagnosis date. Women who did not develop mental illness received a randomly assigned index date and were classified as the second comparison group. We then excluded those $<15$ or $>49$ years at index, with missing postal code data, or with evidence of autoimmune disease before index, in $\geq 1$ physician visits, emergency department visits, or hospitalizations. Women were followed to autoimmune disease diagnosis, emigration, death, or March 31, 2018. In additional analyses, we subdivided women with perinatal mental illness into those diagnosed in pregnancy or $\leq 90$ days postpartum, when most perinatal mental illness cases develop, ${ }^{17}$ and 91-365 days postpartum. We also subdivided women without mental illness into those who were and were not within a perinatal period at index, since pregnancy is a short-term risk factor for autoimmune disease. $^{6}$

\section{Outcome}

The primary outcome was any autoimmune disease, defined by $\geq 1$ emergency department visits or hospitalizations for conditions with a clear autoimmune etiology, using a previously published algorithm. ${ }^{12,18-20}$ We also identified (1) autoimmune disease characterized by brainreactive antibodies, which is most likely associated with mental illness, ${ }^{12}$ and (2) other autoimmune disease, separately (Table S2). We used emergency department visits and hospitalizations to ascertain these outcomes since 3-digit physician visit codes are too broad to distinguish among rare conditions. Thus, we prioritized specificity over sensitivity, capturing the most severe cases but possibly misclassifying those with milder or well-controlled disease, who may only be captured in physician visits.

We also considered two secondary outcome definitions. First, we used a more sensitive, but less specific, definition of any autoimmune disease, in $\geq 2$ physician visits or $\geq 1$ emergency department visits or hospitalizations. Second, we focused on specific autoimmune diseases (ie, Crohn's disease or ulcerative colitis, multiple sclerosis, psoriasis vulgaris, rheumatoid arthritis, and systemic lupus erythematosus) for which there are validated algorithms that use outpatient and acute care data (Table S3) ${ }^{21-25}$ Here, we could be more certain we correctly classified disease status, but only for a smaller list of conditions.

\section{Covariates}

Covariates, measured at index, were age, parity, income quintile, rurality, other chronic conditions, and substance use disorders. For women who were within a perinatal period at index, parity was measured in MOMBABY. For those not within a perinatal period, we searched MOMBABY for deliveries from database inception. If any were identified, we assigned parity as that recorded most proximally to index. Women with no recorded deliveries were assumed to be nulliparous. We measured neighbourhood income quintile by linking postal code with Census data. Rurality was captured by the Rurality Index of Ontario, which uses community factors such as travel time to different care levels to classify neighborhoods as rural (score $\geq 45$ ) or urban $(0-44) .{ }^{26}$ Other chronic conditions were asthma, cardiovascular disease, chronic hypertension, chronic obstructive pulmonary disease, osteoarthritis, and osteoporosis, measured using validated algorithms. $^{27-35}$ Substance use disorder was defined as $\geq 2$ physician visits or $\geq 1$ emergency department visits or hospitalizations for use of illicit drugs or alcohol $<2$ years before index.

\section{Statistical Analyses}

We first used propensity scores to match women with perinatal mental illness to women with mental illness arising outside the perinatal period (the first comparison group). To do this, we used logistic regression to create propensity scores for the probability of perinatal mental illness, with age, parity, income quintile, rurality, other chronic conditions, and substance use disorders as covariates. We used nearest-neighbour greedy matching algorithms based on the propensity score, with a caliper width of 0.20 standard deviations of the logit of the propensity score ${ }^{36}$ and matched women in the exposed to this first comparison group at a ratio of up to $1: 5$, using " $1: n$ matching", which allows a variable matching ratio and has been shown to increase precision in cohort studies. ${ }^{37} \mathrm{We}$ then compared the distributions of covariates in the groups, with standardized differences $<0.10$ showing good balance. ${ }^{38}$ We repeated this process, creating new propensity scores to match the exposed to women without mental illness, at a ratio of up to $1: 3$ for those in a perinatal period at index and 1:5 for those not in a perinatal period at index. Different matching ratios across comparison 
groups were allowed to maximize the sample size in each analysis, and were appropriate since we conducted pairwise comparisons across groups.

We plotted the cumulative probability of autoimmune disease in each group, using Kaplan Meier methods. We used Cox proportional hazards regression to calculate hazard ratios (HR) and 95\% CI for incident autoimmune disease in women with perinatal mental illness compared to women with mental illness arising outside the perinatal period (the first comparison group) and those without mental illness (the second comparison group). A robust sandwich variance estimator accounted for matching. ${ }^{39}$ Women were censored at emigration, death, or March 31, 2018, whichever came first. Mortality during follow-up was rare $(<1 \%)$, suggesting censoring at death was appropriate rather than treating it as a competing risk. ${ }^{40}$ The proportional hazards assumption was tested using Schoenfeld residuals. ${ }^{41}$ Non-proportionality was detected ( $p=0.002)$ with the $\log$-log plot indicating that nonproportionality was confined to the first 90 days of followup, when events were sparse. We used a wash-out period wherein we only examined events $\geq 90$ days after index.

In additional analyses, we repeated the analyses (1) subdividing women with perinatal mental illness by timing of diagnosis, (2) subdividing women without mental illness by whether or not they were in a perinatal period at index, and (3) using the secondary autoimmune disease definitions. We also repeated the analyses excluding women with just one mental illness physician visit from the comparison groups without mental illness. These women did not meet mental illness criteria, but one such visit may signal initial evaluation of suspected mental illness.

Analyses used SAS 9.4 (SAS Institute Inc., Cary, NC).

\section{Results}

\section{Cohort Characteristics}

Between April 1, 1998 and March 31, 2018, we identified 62,764 women with mental illness arising in the perinatal period, of whom 60,701 (96.7\%) were matched with 264,864 women with mental illness arising outside the perinatal period and 469,164 women without mental illness. After matching, all but one standardized difference comparing women with perinatal mental illness to the comparison groups were $<0.10$ (Table 1). Median followup time was 14 years among women with mental illness arising perinatally and those with mental illness arising outside the perinatal period, and 12 years for those without mental illness (also Table 1).

\section{Main Outcomes}

Among women with perinatal mental illness, 1088 (138.4 per 100,000 person-years, 95\% CI 130.2-146.7) developed autoimmune disease, compared with 4778 (140.7 per 100,000 , 95\% CI 136.7-144.7) women with mental illness arising outside the perinatal period and 4950 (88.9 per 100,000, 95\% CI 86.5-91.4) women without mental illness. For all groups, the cumulative probability of autoimmune disease rose most steeply in the first two years of follow-up and rose more slowly thereafter, particularly after 6-8 years of follow-up (Figure 1). The HRs showed the risk of incident autoimmune disease among women with mental illness arising perinatally was similar to that in women with mental illness arising outside the perinatal period (HR 0.98, 95\% CI 0.92-1.05) and higher than that in women without mental illness (HR 1.54, 95\% CI 1.44-1.64) (Table 2).

There were no differences between women with perinatal mental illness and those with mental illness arising outside the perinatal period in the risks of autoimmune disease with brain-reactive antibodies (HR: $1.00,95 \% \mathrm{CI}$ : 0.90-1.12) or other autoimmune disease (HR 0.97, 95\% CI 0.89-1.05). When compared to women without mental illness, risks in women with perinatal mental illness were stronger for autoimmune disease with brain-reactive antibodies (HR 1.71, 95\% CI 1.53-1.90) than for other autoimmune disease (HR 1.45, 95\% CI 1.34-1.58) (Table 2).

\section{Additional Analyses}

When we subdivided women with perinatal mental illness by diagnosis timing and compared them to women with mental illness arising outside the perinatal period, the HR for women diagnosed 91-365 days postpartum was slightly elevated (HR 1.14, 95\% CI 1.03-1.25), whereas the HR for women with perinatal mental illness diagnosed between conception and 90 days postpartum was not elevated. Similarly, compared to women without mental illness, the HR was higher in those with perinatal mental illness diagnosed 91-365 days postpartum (HR 1.78, 95\% CI 1.62-1.96) than those diagnosed between conception and 90 days postpartum (HR 1.40, 95\% CI 1.29-1.52) (Table S4).

When we subdivided women without mental illness into those who were within or not within a perinatal period at index, given the short-term risk of autoimmune disease 
Table I Baseline Characteristics of Women with Mental Illness Arising in the Perinatal Period, Women with Mental Illness Arising Outside the Perinatal Period, and Women Without Mental Illness. Reported as n (\%) Unless Otherwise Indicated

\begin{tabular}{|c|c|c|c|}
\hline \multirow[t]{2}{*}{ Characteristic } & $\begin{array}{c}\text { Mental Illness Arising } \\
\text { Perinatally }\end{array}$ & $\begin{array}{c}\text { Mental Illness Arising Non- } \\
\text { Perinatally }\end{array}$ & No Mental Illness \\
\hline & $(n=60,701)$ & $(n=264,864)$ & $(n=469,164)$ \\
\hline Age (years), mean (SD) & $28.11 \pm 5.94$ & $28.23 \pm 6.69$ & $28.45 \pm 6.16$ \\
\hline $15-19$ & $5440(9.0)$ & $26,880(10.1)$ & $39,621(8.4)$ \\
\hline $20-24$ & $11,769(19.4)$ & $51,693(19.5)$ & $84,232(18.0)$ \\
\hline $25-29$ & I7,588 (29.0) & $70,709(26.7)$ & $|34,68|(28.7)$ \\
\hline $30-34$ & I7,049 (28.1) & $64,264(24.3)$ & $139,225(29.7)$ \\
\hline $35-39$ & $746 \mid(12.3)$ & $44,301(16.7)^{a}$ & $60,307(12.9)$ \\
\hline $40-44$ & $1336(2.2)$ & $6727(2.5)$ & $10,652(2.3)$ \\
\hline $45-49$ & $58(0.1)$ & $290(0.1)$ & $446(0.1)$ \\
\hline \multicolumn{4}{|l|}{ Parity at index date } \\
\hline No previous obstetrical deliveries & $35,605(58.7)$ & $165,464(62.5)$ & $281,830(60.1)$ \\
\hline$\geq$ I previous obstetrical deliveries & $25,096(41.3)$ & $99,400(37.5)$ & $187,334(39.9)$ \\
\hline \multicolumn{4}{|l|}{ Neighbourhood income quintile (Q) } \\
\hline QI (lowest) & $|5| ,47(25.0)$ & $69,463(26.2)$ & II7,597 (25.I) \\
\hline Q2 & $12,676(20.9)$ & $57,536(21.7)$ & 97,101 (20.7) \\
\hline Q3 & $12,339(20.3)$ & $53,199(20.1)$ & $94,983(20.2)$ \\
\hline Q4 & $11,562(19.0)$ & $46,719(17.6)$ & $89,144(19.0)$ \\
\hline Q5 (highest) & $8977(14.8)$ & $37,947(14.3)$ & $70,339(15.0)$ \\
\hline Rural residence & $6980(11.5)$ & $30,182(11.4)$ & $52,783(11.3)$ \\
\hline $\begin{array}{l}\text { Cardiovascular disease or } \\
\text { hypertension }\end{array}$ & $1054(1.7)$ & $5162(1.9)$ & $7980(1.7)$ \\
\hline Chronic respiratory disease ${ }^{b}$ & $5653(9.3)$ & $26,020(9.8)$ & $38,887(8.3)$ \\
\hline Musculoskeletal disorders ${ }^{c}$ & $545(0.9)$ & $2610(1.0)$ & $4086(0.9)$ \\
\hline Substance use disorder & $282(0.5)$ & $1562(0.6)$ & $1935(0.4)$ \\
\hline Mean follow-up, years (SD) & $13.0(5.4)$ & $12.8(5.5)$ & $11.9(5.8)$ \\
\hline Median follow-up, years (range) & $14(0-2 \mid)$ & $14(0-2 \mid)$ & $12(0-2 \mid)$ \\
\hline
\end{tabular}

Notes: ${ }^{\mathrm{S}}$ Standardized difference was $>0.10$ when compared to women with perinatal mental illness. ${ }^{\mathrm{b}}$ Includes asthma and chronic obstructive pulmonary disease. ${ }^{\mathrm{c}}$ Includes osteoarthritis and osteoporosis.

associated with pregnancy, HRs comparing women with perinatal mental illness to these two groups were similar to each other (perinatal HR 1.54, 95\% CI 1.43-1.66; nonperinatal HR 1.54, 95\% CI 1.43-1.65) (Figure S1, Table S5).

When we used the secondary outcome definitions, HRs were slightly attenuated when comparing women with perinatal mental illness to those without mental illness; similar to the main analysis, there were no differences between women with perinatal vs non-perinatal onset of mental illness (Table S6).

When we removed women with just one mental illness physician visit from the comparison groups without mental illness, HRs were slightly stronger than in the main analysis (Table S7).

\section{Discussion}

In this large, population-based study, we found that women with mental illness arising perinatally were at elevated risk of autoimmune disease, and particularly autoimmune disease with brain-reactive antibodies, compared to those without a mental illness. No meaningful differences in risk were observed compared to women with mental illness arising outside the perinatal period. Risks were robust in additional analyses, though for mental illness arising in the late postpartum period, slightly elevated 

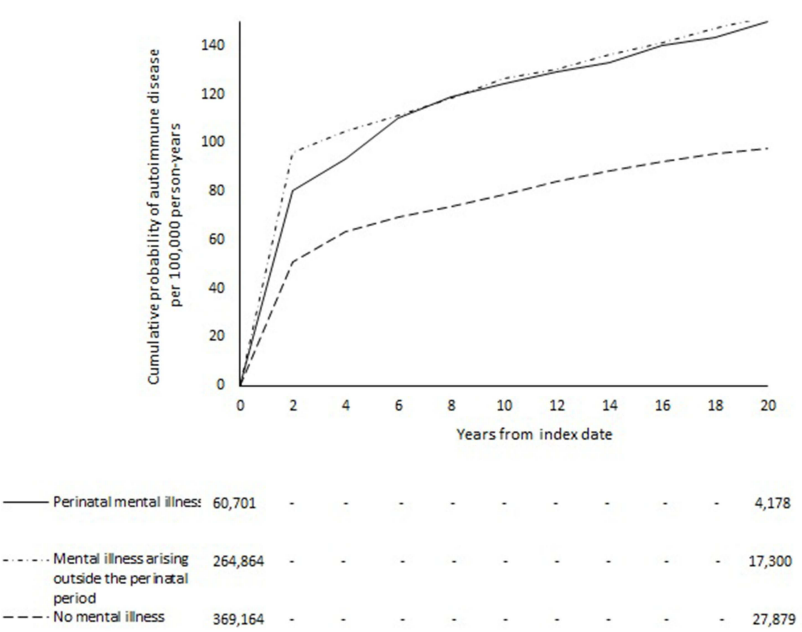

Figure I Cumulative probability of autoimmune disease after 90 days among women with mental illness arising in the perinatal period, women with mental illness arising outside the perinatal period, and women without mental illness. The median (interquartile range) time-to-event overall, and among those with the event, was $13.7(8.9-17.6)$ and $8.3(4.6-12.6)$ years for those with mental illness arising in the perinatal period; $13.6(8.6-17.6)$ and $8.4(4.5-12.8)$ years for those with mental illness arising outside the perinatal period; and 12.3 (7.0-17.0) and 8.3 (4.4-12.4) years for those without mental illness.

risk was observed compared to women with mental illness arising non-perinatally. Overall, these findings suggest women with mental illness are at risk for autoimmune disease regardless of the timing of onset of mental illness -ie, that women with mental illness arising perinatally are at no greater risk than their peers with mental illness arising outside the perinatal period, but also that they are no less at risk.

Our results are consistent with studies showing women with perinatal mental illness are at elevated risk for autoimmune disease. In a population-based cohort of 2707 primiparas in Taiwan, Lin et $\mathrm{al}^{4}$ showed women with postpartum depression had increased risk, compared to those without postpartum depression, for autoimmune disease (aHR 1.61, 95\% CI 1.30-1.99). Likewise, among 148 Dutch women, Bergink et $\mathrm{al}^{5}$ found women with postpartum psychosis had a higher rate of autoimmune thyroid disease than their peers (19\% vs 5\%). Until now, however, the extent to which this association is uniquely related to mental illness arising perinatally has been unclear, since prior studies excluded women with mental illness arising outside the perinatal period. ${ }^{4,5}$ Indeed, in the general population, studies have demonstrated higher risk of autoimmune disease in adults with versus without mental illness. ${ }^{11}$ Our study adds to the literature by including women with mental illness arising perinatally and outside the perinatal period, as well as women without mental illness, to disentangle these effects.

The risk of autoimmune disease associated with mental illness may be due to the impact of chronic distress on the sympathetic nervous system and hypothalamic-pituitaryadrenal axis, both of which regulate immune functioning. ${ }^{13,14}$ Immune-mediated cytokine alterations have been found in adults with mental illness, ${ }^{42}$ including women with perinatal depression. ${ }^{9,10}$ Also, brain-reactive antibodies, found in $2-3 \%$ of the population, contribute to brain pathology and autoimmunity. ${ }^{7,12}$ Some studies ${ }^{6}$ have posited that perinatal mental illness is an autoimmune condition because it flares in early pregnancy and early postpartum, similar to diseases such as rheumatoid arthritis that are affected by the immunologic response to implantation and delivery. ${ }^{8}$ While our data do not support an association that is specific to mental illness arising perinatally, "onset" of mental illness was based on diagnosis date, which may be affected by barriers accessing health care. ${ }^{43}$ This could have masked nuances related to mental illness timing; for example, a delay between mental illness onset and diagnosis could explain why mental illness in the late postpartum period, not early postpartum, ${ }^{6}$ had a slightly stronger impact on autoimmune disease risk. Nevertheless, given evidence of the role of immune functioning in mental illness and autoimmune disease, a broad association between the two disorders is plausible.

Clinically, our findings suggest women with mental illness should be monitored for autoimmune disease. There is growing understanding of the need for early detection of metabolic and cardiovascular disease in persons with mental illness; ${ }^{44}$ our data suggest autoimmune disease is also important to consider. The absence of a difference in risk for autoimmune disease between women with mental illness arising perinatally vs outside the perinatal period is also important. Perinatal mental illness has been considered a time-limited condition that does not necessarily recur, with long-term impacts presumed to be less significant than those of mental illness outside the perinatal period. However, our data suggest a history of mental illness in pregnancy or postpartum should be taken just as seriously as a risk factor for chronic disease as mental illness outside the perinatal period. Our findings further indicate the need for more research on the potential common etiology of mental illness and autoimmune disease. This is particularly important for reproductive-aged women, since autoimmunity has 
Table 2 Risk of Incident Autoimmune Disease After 90 Days Among Women with Mental Illness Arising in the Perinatal Period Compared Separately to: Women with Mental Illness Arising Outside the Perinatal Period and Women Without Mental Illness

\begin{tabular}{|c|c|c|c|c|c|c|}
\hline \multirow[t]{2}{*}{ Outcome } & \multirow[t]{2}{*}{ Groups Being Compared } & \multirow[t]{2}{*}{$\begin{array}{c}\text { Number (\%) with } \\
\text { Outcome Event }\end{array}$} & \multicolumn{2}{|c|}{$\begin{array}{l}\text { Incidence Rate per } \\
\text { 100,000 Person-Years }\end{array}$} & \multicolumn{2}{|c|}{ Model $^{\mathrm{a}}$} \\
\hline & & & $\begin{array}{l}\text { Incidence } \\
\text { Rate }\end{array}$ & $95 \% \mathrm{Cl}$ & HR & $95 \% \mathrm{Cl}$ \\
\hline \multirow[t]{4}{*}{ Any autoimmune disease } & $\begin{array}{l}\text { Mental illness arising in the } \\
\text { perinatal period }\end{array}$ & $1088(1.8)$ & 138.4 & $130.2-146.7$ & 0.98 & $0.92-1.05$ \\
\hline & $\begin{array}{l}\text { Mental illness arising outside } \\
\text { the perinatal period }\end{array}$ & $4778(1.8)$ & 140.7 & |36.7-|44.7 & 1.00 & Referent \\
\hline & $\begin{array}{l}\text { Mental illness arising in the } \\
\text { perinatal period }\end{array}$ & $1088(1.8)$ & 138.4 & $\mid 30.2-146.7$ & 1.54 & $1.44-1.64$ \\
\hline & No mental illness & $4950(1.1)$ & 88.9 & $86.5-91.4$ & 1.00 & Referent \\
\hline \multirow[t]{4}{*}{$\begin{array}{l}\text { Autoimmune disease with } \\
\text { brain-reactive antibodies }\end{array}$} & $\begin{array}{l}\text { Mental illness arising in the } \\
\text { perinatal period }\end{array}$ & $403(0.7)$ & 51.0 & $46.0-56.0$ & 1.00 & $0.90-1.12$ \\
\hline & $\begin{array}{l}\text { Mental illness arising outside } \\
\text { the perinatal period }\end{array}$ & $1735(0.7)$ & 50.8 & $48.4-53.2$ & 1.00 & Referent \\
\hline & $\begin{array}{l}\text { Mental illness arising in the } \\
\text { perinatal period }\end{array}$ & $403(0.7)$ & 51.0 & $46.0-56.0$ & 1.71 & $1.53-1.90$ \\
\hline & No mental illness & $1642(0.4)$ & 29.4 & $28.0-30.8$ & 1.00 & Referent \\
\hline \multirow[t]{4}{*}{ Other autoimmune disease } & $\begin{array}{l}\text { Mental illness arising in the } \\
\text { perinatal period }\end{array}$ & $708(1.2)$ & 89.8 & $83.2-96.4$ & 0.97 & $0.89-1.05$ \\
\hline & $\begin{array}{l}\text { Mental illness arising outside } \\
\text { the perinatal period }\end{array}$ & $3153(1.2)$ & 92.6 & $89.4-95.8$ & 1.00 & Referent \\
\hline & $\begin{array}{l}\text { Mental illness arising in the } \\
\text { perinatal period }\end{array}$ & $708(1.2)$ & 89.8 & $83.2-96.4$ & 1.45 & $1.34-1.58$ \\
\hline & No mental illness & $3404(0.7)$ & 61.0 & $59.0-63.1$ & 1.00 & Referent \\
\hline
\end{tabular}

Note: ${ }^{\mathrm{a}}$ Groups were matched on age, parity, neighbourhood income quintile, area of residence, other chronic conditions, and substance use disorders.

implications for fertility and pregnancy loss, ${ }^{45,46}$ and pregnancy itself can affect autoimmunity. ${ }^{47}$

Our study design was built on the hypothesis that it is the nature of mental illness onset (ie, perinatal versus nonperinatal) that may confer unique risk for autoimmune disease. By excluding women with a history of mental illness before index, we aimed to capture new-onset mental illness. However, for a small proportion of women, we may have missed diagnoses occurring before database inception (1988) or migration to Ontario. Some mental illness may go undetected by the health system, resulting in potential exposure misclassification. We were unable to measure mental illness severity, which could impact autoimmune disease risk, and we did not examine the risk of autoimmune disease by type of mental illness. However, prior studies have shown relationships between psychotic, mood and anxiety, and other mental disorders and autoimmune disease. ${ }^{11,12,19,20}$ We could not measure the occurrence of "baby blues" or "postpartum blues", which describe mood disturbances that are common within 2-4 days after childbirth and typically resolve within 14 days. ${ }^{48}$ However, due to the transient nature of baby blues compared to postpartum depression and other types of perinatal mental illness, ${ }^{48}$ we would not expect baby blues to increase the risk of autoimmune disease. We measured 29 of the most common autoimmune diseases using a previously published algorithm. ${ }^{18-20}$ However, there are other, rare, autoimmune conditions that were not captured. Our primary definition of autoimmune disease was limited by the inability to capture diagnoses in 
outpatient settings, potentially missing mild or wellcontrolled disease. However, findings were robust to the use of more sensitive outcome definitions. We did not capture data on the role of medications in the observed association, and were unable to control for smoking and obesity, which are associated with mental illness ${ }^{49,50}$ and autoimmune disease. ${ }^{51,52}$ Finally, median follow-up was slightly shorter in women without mental illness; however, the small difference compared to the exposed (2 years) was small.

\section{Conclusions}

Perinatal mental illness is associated with increased risk of autoimmune disease that is no different than that of mental illness arising non-perinatally. Women with mental illness, regardless of the timing of onset, could benefit from early detection of autoimmune disease.

\section{Data Sharing Statement}

Data used for this study were housed at ICES, an independent not-for-profit corporation. While data sharing agreements prohibit ICES from making the data set publicly available, access can be granted to those who to meet prespecified criteria for confidential access, available at www. ices.on.ca/. Requests to access ICES data for research purposes may be submitted to ICES' Data and Analytic Services. Visit http://www.ices.on.ca/DAS for more information, including contact details.

\section{Ethics Approval and Informed Consent}

ICES is a prescribed entity under Ontario's Personal Health Information Protection Act (PHIPA). Section 45 of PHIPA authorizes ICES to collect personal health information, without consent, for the purpose of analysis or compiling statistical information with respect to the management of, evaluation or monitoring of, the allocation of resources to or planning for all or part of the health system. Projects that use data collected by ICES under section 45 of PHIPA, and use no other data, are exempt from REB review. The use of the data in this project is authorized under section 45 and approved by ICES' Privacy and Legal Office.

\section{Acknowledgments}

This study was supported by ICES, which is funded by an annual grant from the Ontario Ministry of Health (MOH) and Ministry of Long-Term Care (MLTC). The opinions, results and conclusions reported in this paper are those of the authors and are independent from the funding sources. No endorsement is intended or should be inferred. Parts of this material are based on data and information compiled and provided by the Canadian Institute for Health Information (CIHI). However, the analyses, conclusions, opinions, and statements expressed herein are those of the author, and not necessarily those of CIHI.

\section{Author Contributions}

Hilary K. Brown undertook the conception and design of the study, the analysis and interpretation of the data, and drafting of the manuscript. Andrew Wilton undertook the analysis and interpretation of the data and critically revised the manuscript for important intellectual content. Ning Liu, Joel G. Ray, Cindy-Lee Dennis, and Simone Vigod contributed to the conception and design of the study and the interpretation of the data and critically revised the manuscript for important intellectual content. All of the authors gave final approval of the version to be published; agreed on the journal to which the article has been submitted; and agree to be accountable for all aspects of the work.

\section{Funding}

We gratefully acknowledge the Canadian Institutes of Health Research for their support of this study through their Project Grant program (376290). This research was undertaken, in part, thanks to funding from the Canada Research Chairs Program. The funding agency had no role in the design and conduct of the study; collection, management, analysis, and interpretation of the data; preparation, review, or approval of the manuscript; or decision to submit the manuscript for publication.

\section{Disclosure}

Dr. Vigod receives royalties from UpToDate Inc for authorship of materials related to depression and pregnancy. All other authors report no conflicts of interest.

\section{References}

1. O’Hara MW, Wisner KL. Perinatal mental illness: definition, description and aetiology. Best Pract Res Clin Obstet Gynaecol. 2014;28 (1):3-12. doi:10.1016/j.bpobgyn.2013.09.002

2. Meltzer-Brody S, Stuebe A. The long-term psychiatric and medical prognosis of perinatal mental illness. Best Pract Res Clin Obstet Gynaecol. 2014;28(1):49-60. doi:10.1016/j.bpobgyn.2013.08.009

3. Deave T, Heron J, Evans J, Emond A. The impact of maternal depression in pregnancy on early child development. BJOG. 2008;115 (8):1043-1051. doi:10.1111/j.1471-0528.2008.01752.x 
4. Lin CY, Li CK, Liu JM, Hsu RJ, Chuang HC, Chang FW. Postpartum depression and subsequent autoimmune diseases in Taiwan. Int $J$ Environ Res Public Health. 2018;15(8):20. doi:10.3390/ ijerph15081783

5. Bergink V, Kushner SA, Pop V, et al. Prevalence of autoimmune thyroid dysfunction in postpartum psychosis. $\mathrm{Br} J$ Psychiatry. 2011;198(4):264-268. doi:10.1192/bjp.bp.110.082990

6. Gleicher N. Postpartum depression, an autoimmune disease? Autoimmun Rev. 2007;6(8):572-576. doi:10.1016/j.autrev.2007.04.002

7. Diamond B, Honig G, Mader S, Brimberg L, Volpe B. Brain-reactive antibodies and disease. Аnпи Rev Immunol. 2013;31:345-385. doi:10.1146/annurev-immunol-020711-075041

8. Adams Waldorf KM, Nelson JL. Autoimmune disease during pregnancy and the microchimerism legacy of pregnancy. Immunol Invest. 2008;37(5-6):631-644. doi:10.1080/08820130802205886

9. Maes M, Lin A-H, Ombelet W, et al. Immune activation in the early puerperium is related to postpartum anxiety and depressive symptoms. Psychoneuroendocrinology. 2000;25(2):121-137. doi:10.1016/S0306-4530(99)00043-8

10. Boufidou F, Lambrinoudaki I, Argeitis J, et al. CSF and plasma cytokines at delivery and postpartum mood disturbances. $J$ Affect Disord. 2009;115(1-2):287-292. doi:10.1016/j.jad.2008.07.008

11. Andersson N, Gustafsson L, Okkels N, et al. Depression and the risk of autoimmune disease: a nationally representative, prospective longitudinal study. Psych Med. 2015;45(16):3559-3569. doi:10.1017/ S0033291715001488

12. Benros ME, Waltoft BL, Nordentoft M, et al. Autoimmune diseases and severe infections as risk factors for mood disorders: a nationwide study. JAMA Psychiatry. 2013;70(8):812-820. doi:10.1001/ jamapsychiatry.2013.1111

13. Irwin MR, Cole SW. Reciprocal regulation of the neural and innate immune systems. Nat Rev Immunol. 2011;11(9):625. doi:10.1038/nri3042

14. Miller AH, Maletic V, Raison CL. Inflammation and its discontents: the role of cytokines in the pathophysiology of major depression. Biol Psychiatry. 2009;65(9):732-741. doi:10.1016/j.biopsych.2008.11.029

15. Williams JI, Young W. Summary of studies on the quality of health care administrative databases in Canada. In: Goel VW, editor. Patterns of Health Care in Ontario, the ICES Practice Atlas. Toronto: Canadian Medical Association; 1996.

16. You JJ, Alter DA, Stukel TA, et al. Proliferation of prenatal ultrasonography. CMAJ. 2010;182(2):143-151. doi:10.1503/cmaj.090979

17. Munk-Olsen T, Maegbaek M, Johannsen B, et al. Perinatal psychiatric episodes: a population-based study on treatment incidence and prevalence. Transl Psychiatry. 2016;6(10):e919. doi:10.1038/tp.2016.190

18. Eaton WW, Rose NR, Kalaydjian A, Pedersen MG, Mortensen PB. Epidemiology of autoimmune diseases in Denmark. J Autoimmun. 2007;29(1):1-9. doi:10.1016/j.jaut.2007.05.002

19. Eaton WW, Byrne M, Ewald H, et al. Association of schizophrenia and autoimmune diseases: linkage of Danish national registers. $A m$ J Psychiatry. 2006;163(3):521-528. doi:10.1176/appi.ajp.163.3.521

20. Eaton WW, Pederson MG, Nielsen PR, Mortensen PB. Autoimmune disease, bipolar disorder, and non-affective psychosis. Bipolar Disord. 2010;12(6):638-646. doi:10.1111/j.1399-5618.2010.00853.x

21. Benchimol EI, Guttmann A, Mack DR, et al. Validation of international algorithms to identify adults with inflammatory bowel disease in health administrative data from Ontario, Canada. J Clin Epidemiol. 2014;67(8):887-896. doi:10.1016/j.jclinepi.2014.02.019

22. Widdifield J, Ivers NM, Young J, et al. Development and validation of an administrative data algorithm to estimate the disease burden and epidemiology of multiple sclerosis in Ontario, Canada. Mult Scler. 2015;21(8):1045-1054. doi:10.1177/1352458514556303

23. Widdifield J, Bombardier C, Bernatsky S, et al. An administrative data validation study of the accuracy of algorithms for identifying rheumatoid arthritis: the influence of the reference standard on algorithm performance. BMC Musculoskelet Disord. 2014;15(1):216. doi:10.1186/1471-2474-15-216
24. Bernatsky S, Joseph L, Pineau C, Tamblyn R, Feldman D, Clarke A. A population-based assessment of systemic lupus erythematosus incidence and prevalence - results and implications of using administrative data for epidemiological studies. Rheumatology. 2007;46 (12):1814-1818. doi:10.1093/rheumatology/kem233

25. Eder L. Accuracy of Canadian administrative health data in identifying ptients with psoriasis and psoriatic arthritis using primary care medical records as the reference standard. American College of Rheumatology Annual Meeting; 2017.

26. Kralj B. Measuring "rurality" for purposes of health-care planning: an empirical measure for Ontario. Ont Med Rev. 2000;67(9):33-52.

27. Austin PC, Daly PA, Tu JV. A multicenter study of the coding accuracy of hospital discharge administrative data for patients admitted to cardiac care units in Ontario. Am Heart J. 2002;144 (2):290-296. doi:10.1067/mhj.2002.123839

28. Gershon AS, Wang C, Guan J, Vasilevska-Ristovska J, Cicutto L, To T. Identifying patients with physician-diagnosed asthma in health administrative databases. Can Respir J. 2009;16(6):183-188.

29. Tu K, Nieuwlaat R, Cheng SY, et al. Identifying patients with atrial fibrillation in administrative data. Can $J$ Cardiol. 2016;32 (12):1561-1565. doi:10.1016/j.cjca.2016.06.006

30. Gershon A, Wang C, Guan J, Vasilevska-Ristovska J, Cicutto L, To T. Identifying individuals with physcian diagnosed COPD in health administrative databases. COPD. 2009;6(5):388-394. doi:10.1080/ 15412550903140865

31. Schultz S, Rothwell D, Chen Z, Tu K. Identifying cases of congestive heart failure from administrative data: a validation study using primary care patient records. Chronic Dis Inj Can. 2013;33(3):160-166. doi:10.24095/hpcdp.33.3.06

32. Hux JE, Ivis F, Flintoft V, Bica A. Diabetes in Ontario: determination of prevalence and incidence using a validated administrative data algorithm. Diabetes Care. 2002;25(3):512-516. doi:10.2337/ diacare.25.3.512

33. Tu K, Campbell NR, Chen Z-L, Cauch-Dudek KJ, McAlister FA. Accuracy of administrative databases in identifying patients with hypertension. Open Med. 2007;1(1):e18.

34. Fleet JL, Dixon SN, Shariff SZ, et al. Detecting chronic kidney disease in population-based administrative databases using an algorithm of hospital encounter and physician claim codes. BMC Nephrol. 2013;14(1):81. doi:10.1186/1471-2369-14-81

35. Tu K, Wang M, Young J, et al. Validity of administrative data for identifying patients who have had a stroke or transient ischemic attack using EMRALD as a reference standard. Can J Cardiol. 2013;29(11):1388-1394. doi:10.1016/j.cjca.2013.07.676

36. Austin PC. Optimal caliper widths for propensity-score matching when estimating differences in means and differences in proportions in observational studies. Pharmaceutical Stay. 2011;10(2):150-161. doi: $10.1002 /$ pst.433

37. Rassen JA, Shelat AA, Myers J, Glynn RJ, Rothman KJ, Schneeweiss S. One-to-many propensity score matching in cohort studies. Pharmacoepidemiol Drug Saf. 2012;21:69-80. doi:10.1002/pds.3263

38. Austin PC. Using the standardized difference to compare the prevalence of a binary variable between two groups in observational research. Comm Stat Sim Comp. 2009;38(6):1228-1234. doi:10.1080/03610910902859574

39. Austin PC. The performance of different propensity score methods for estimating marginal hazard ratios. Stat Med. 2013;32 (16):2837-2849. doi:10.1002/sim.5705

40. Austin PC, Lee DS, Fine JP. Introduction to the analysis of survival data in the presence of competing risks. Circulation. 2016;133 (6):601-609. doi:10.1161/CIRCULATIONAHA.115.017719

41. Schoenfeld D. Partial residuals for the proportional hazards regression model. Biometrika. 1982;69(1):239-241. doi:10.1093/biomet/69.1.239

42. Pucak ML, Kaplin AI. Unkind cytokines: current evidence for the potential role of cytokines in immune-mediated depression. Int Rev Psychiatry. 2005;17(6):477-483. doi:10.1080/02646830500381757 
43. Coates AO, Schaefer CA, Alexander JL. Detection of postpartum depression and anxiety in a large health plan. J Behav Health Serv Res. 2004;31(2):117-133. doi:10.1007/BF02287376

44. De Hert M, Dekker J, Wood D, Kahl K, Holt R, Möller H-J. Cardiovascular disease and diabetes in people with severe mental illness position statement from the European Psychiatric Association (EPA), supported by the European Association for the Study of Diabetes (EASD) and the European Society of Cardiology (ESC). Eur Psychiatry. 2009;24(6):412-424. doi:10.1016/j.eurpsy.20 09.01 .005

45. Kutteh W. Autoimmune factors in assisted reproduction. Minerva Ginecol. 2002;54(3):217-224.

46. Carp H, Meroni P, Shoenfeld Y. Autoantibodies as predictors of pregnancy complications. Rheumatology. 2008;47(S3):6-8. doi:10.1093/rheumatology/ken154

47. Cervera R, Balasch J. Bidirectional effects on autoimmunity and reproduction. Hum Reprod Update. 2008;14(4):359-366. doi:10.10 93/humupd/dmn013

48. Beck CT, Reynolds MA, Rutowski P. Maternity blues and postpartum depressoin. J Obstet Gynecol Neonatal Nurs. 1992;21(4):287-293. doi:10.1111/j.1552-6909.1992.tb01739.x
49. Taylor VH, McIntyre RS, Remington G, Levitan RD, Stonehocker B, Sharma AM. Beyond pharmacotherapy: understanding the links between obesity and chronic mental illness. Can J Psychiatry. 2012;57(1):5-12. doi:10.1177/070674371205700103

50. Bakhshaie J, Zvolensky MJ, Goodwin RD. Cigarette smoking and the onset and persistence of depression among adults in the United States: 1994-2005. Compr Psychiatry. 2015;60:142-148. doi:10.10 16/j.comppsych.2014.10.012

51. Versini M, Jeandel P-Y, Rosenthal E, Shoenfeld Y. Obesity in autoimmune diseases: not a passive bystander. Autoimmun Rev. 2014;13 (9):981-1000

52. van der Heide F, Wassenaar M, van der Linde K, Spoelstra P, Kleibeuker JH, Dijkstra G. Effects of active and passive smoking on crohn's disease and ulcerative colitis in a cohort from a regional hospital. Eur J Gastroenterol Hepatol. 2011;23(3):255-261. doi:10.1097/MEG.0b013e3283435233
Clinical Epidemiology

\section{Publish your work in this journal}

Clinical Epidemiology is an international, peer-reviewed, open access, online journal focusing on disease and drug epidemiology, identification of risk factors and screening procedures to develop optimal preventative initiatives and programs. Specific topics include: diagnosis, prognosis, treatment, screening, prevention, risk factor modification,

\section{Dovepress}

systematic reviews, risk \& safety of medical interventions, epidemiology \& biostatistical methods, and evaluation of guidelines, translational medicine, health policies \& economic evaluations. The manuscript management system is completely online and includes a very quick and fair peer-review system, which is all easy to use. 